\title{
Aggregation of liposomes induced by calcium: A structural and kinetic study
}

\author{
Sándalo Roldán-Vargas, ${ }^{1}$ Alberto Martín-Molina, ${ }^{1}$ Manuel Quesada-Pérez, ${ }^{2}$ Ramon Barnadas-Rodríguez, ${ }^{3}$ \\ Joan Estelrich, ${ }^{3}$ and José Callejas-Fernández ${ }^{1, *}$ \\ ${ }^{1}$ Grupo de Física de Fluidos y Biocoloides, Departamento de Física Aplicada, Universidad de Granada, E-18071 Granada, Spain \\ ${ }^{2}$ Departamento de Física, Escuela Politécnica Superior de Linares, Universidad de Jaén, Linares, E-23700 Jaén, Spain \\ ${ }^{3}$ Unitat de Fisicoquímica, Facultat de Farmàcia, Universitat de Barcelona, E-08028 Barcelona, Catalonia, Spain
}

(Received 9 October 2006; revised manuscript received 27 November 2006; published 21 February 2007)

In this work, the calcium-induced aggregation of phosphatidylserine liposomes is probed by means of the analysis of the kinetics of such process as well as the aggregate morphology. This novel characterization of liposome aggregation involves the use of static and dynamic light-scattering techniques to obtain kinetic exponents and fractal dimensions. For salt concentrations larger than $5 \mathrm{mM}$, a diffusion-limited aggregation regime is observed and the Brownian kernel properly describes the time evolution of the diffusion coefficient. For slow kinetics, a slightly modified multiple contact kernel is required. In any case, a time evolution model based on the numerical resolution of Smoluchowski's equation is proposed in order to establish a theoretical description for the aggregating system. Such a model provides an alternative procedure to determine the dimerization constant, which might supply valuable information about interaction mechanisms between phospholipid vesicles.

DOI: 10.1103/PhysRevE.75.021912

PACS number(s): 87.68.+z, 87.83.+a

\section{INTRODUCTION}

Phospholipid vesicles (liposomes) are colloidal systems that present a major interest in the pharmaceutical, cosmetic, and food industry since they are biocompatible structures to encapsulate proteins, nucleic acids, drugs, etc. Moreover, liposomes are considered as model systems of the cellular membrane. In particular, they have been widely applied to the study of biological transport through membranes, induced aggregation phenomena, etc...[1-3]. Therefore, liposomes play an important role in many research areas since the early 60s. Besides their numerous medical applications, liposomes could be considered as model colloidal systems since magnitudes such as size, charge, and rigidity can be easily controlled during their synthesis. In this sense, vesicular suspensions present the typical properties of colloidal dispersions from a physicochemical point of view. This twofold role of liposomes is precisely the main advantage of this system. Namely, a colloidal formalism of aggregation applied to phospholipid vesicles appears as a crucial step for a better understanding of complex phenomena in cell biology, such as the membrane fusion. In this sense, knowledge about the temporal evolution of an aggregating colloidal system, as well as the study of the geometrical properties of the resulting aggregates, have been demonstrated to be a key to understand and control the interaction mechanism between individual particles.

Due to the importance of the aggregation-fusion between vesicles, in the last decades considerable progress has been made toward the comprehension of the underlying mechanisms and factors involved in these processes $[1,4-6]$. In most of these works, it is well established that the ioninduced vesicular aggregation and fusion depend essentially on the composition, charge, size, and concentrations of the

\footnotetext{
*Email address: jcalleja@ugr.es
}

liposomes, as well as on characteristics of the dispersion media such as $\mathrm{pH}$, ionic strength, temperature, and type of ions presented therein. In particular, the well-known fact that $\mathrm{Ca}^{2+}$ is involved in many biological membrane fusion phenomena results in numerous research works devoted to the study of aggregation and fusion of pure bovine brain phosphatidylserine (PS) liposomes by effect of the divalent cations $[4,5,7,8]$. Accordingly, the main goal of this research work is dedicated to deepen into the study of the $\mathrm{Ca}^{2+}$-induced aggregation between PS liposomes by means of a novel modus operandi based on the analysis of the aggregation kinetics as well as the morphology of the resulting aggregates.

From the point of view of colloidal science, an enormous effort has been done in the past in order to reach a whole knowledge of the particle aggregation phenomena from theoretical, simulation, and experimental approaches. However, in reviewing the literature, the aggregation of "biological particles" such proteins or vesicles has been scarcely studied using the well-developed techniques from colloidal aggregation. This is the main interest of this work, to describe the aggregation of vesicles using some methods from colloidal science. This is the primal reason why in this work the aggregation kinetics and cluster structure of the liposomes are analyzed. On one hand, the term cluster structure refers to the spatial distribution of the monomers into the aggregate. When a scale-invariant growth appears the geometrical description can be expressed in terms of a cluster fractal dimension, $d_{f}$. In colloidal science, this magnitude can be straightforwardly determined by means of scattering techniques; hence it is surprising that this magnitude has been rarely employed in the studies of aggregation of liposomes as Lasic claims [7]. Certainly, there are previous works in which liposomes and their aggregation processes are studied by light scattering. However, the number of them that apply the notion of fractal dimension is considerably scarce. For instance, Bordi et al. [9] offers an advisable work in which light scattering becomes a powerful tool to investigate polyion- 
induced aggregation but fractal dimensions are not considered by them. Stauch and Schubert [10] do apply this concept to the study of the inner structure of chemically modified liposomes (but as individual entities). As far as we know, only Lynch et al. looked into the kinetics of liposome aggregation (induced by proteins in such case) by means of turbidity measurements with the help of this concept [11].

On the other hand, aggregation kinetics addresses the possibility of doing a whole description of the aggregating dispersion, i.e., to know at a fixed time the number of clusters $N_{n}(t)$ having $n$ particles for each one. A feasible approach to solving this nontrivial problem is the resolution of the Smoluchowski equation at which the key parameters are the "aggregation kernels", i.e., a set of kinetic rate constants for all possible cluster-cluster reactions. Additionally, the van Dongen-Ernst homogeneity parameter, $\lambda$, can be useful to complete the description of an aggregating system $[12,13]$.

Historically, two limiting regimes have been identified in colloidal aggregation: a rapid diffusion-limited cluster aggregation (DLCA) and a slow reaction-limited cluster aggregation (RLCA). Salt-induced aggregation of electrically stabilized bare particles aggregating at low and high electrolyte concentration is an example where these limiting regimes have been successfully obtained. To date, both the resolution of Smoluchowski's equation (via the selection of an adequate kernel) and the kinetics description in the van Dongen-Ernst scheme have not been applied to liposome aggregation.

Bearing all this in mind, the paper is organized as follows: First, an overview of the colloidal aggregation theory is presented. Next, a section in which the experimental procedures are described. Finally, experimental and numerical results are shown. Namely, the spatial distribution of liposomes into the clusters is estimated via measurements of the aggregate fractal dimension obtained by means of static light scattering (SLS). On the other hand, dynamic light scattering (DLS) technique is used to describe the kinetics of the liposome aggregation induced by calcium. In addition, an evolution kinetic model based in the numerical resolution of Smoluchowski's equation is proposed in order to establish a theoretical description for the aggregating system.

\section{THEORETICAL BACKGROUND}

\section{A. Fractal geometry and static light scattering}

The spatial correlations between particles of a structure are usually accounted for the pair correlation function $c(\vec{r})$,

$$
c(\vec{r})=\frac{1}{V} \sum_{r^{\prime}} \rho\left(\vec{r}+\vec{r}^{\prime}\right) \rho\left(\vec{r}^{\prime}\right),
$$

where $V$ is the volume of the structure and $\rho(\vec{r})$ the local density $[\rho(\vec{r})=1$ if $\vec{r}$ belongs to the structure, otherwise it is equal to zero]. The functional behavior of $c(\vec{r})$ can be used as a criterion to determine the fractal growth of an object (aggregate). An isotropic finite object presents a nontrivial scale-invariant growth if [14]

$$
c(r) \propto r^{d_{f}-d} f\left(r / R_{o b j}\right),
$$

where $d$ is the topological spatial dimension, $d_{f}$ is the fractal dimension, $R_{o b j}$ the average radius of the object, and $f(x)$ is a cutoff function in which the finite size of the object is considered, satisfying $f(x) \approx 1$ for $x \ll 1$ and $f(x) \approx 0$ for $x \gg 1$.

According to its definition in scattering theory, the structure factor $S(q)$ is related with the pair correlation function through a Fourier transform [14]. This relationship gives an equivalent condition for (2), expressed via $S(q)$,

$$
S(q) \propto q^{-d_{f}}\left(1 / R_{o b j} \ll q \ll 1 / a\right),
$$

where the finite size of the constituent particles of the object has been considered through the domain given by the inequality, $\vec{q}=\vec{k}_{i}-\vec{k}_{s}$ is the scattering vector, defined as the difference between the incident and the scattered wave vectors, and $a$ is the average radius of the particles.

Thanks to this equivalent description in the $q$ space given by the structure factor, the fractal geometry of a distribution of aggregates in a three-dimensional (3D) suspension can be determined by means of an SLS experiment, where experimental values for $S(q)$ and, in the case that fractal nature appears, $d_{f}$, are obtained. This experimental access is given by

$$
S(q)=\frac{\rho_{0}\langle I(q)\rangle}{\rho\left\langle I_{0}(q)\right\rangle},
$$

where we assume $q=\left(4 \pi / \lambda_{m}\right) \sin (\theta / 2)$ (elastic light scattering), $\lambda_{m}$ being the wavelength of the incident light beam in the medium, $\theta$ is the scattering angle, $\rho$ is the particle concentration, and $\langle I(q)\rangle$ is the time-averaged light intensity scattered by the particle dispersion. The lower index ${ }_{0}$ indicates the corresponding values to the diluted and disordered suspension.

\section{B. Diffusion and dynamic light scattering (DLS)}

The temporal fluctuations in the instantaneous electric field, $E(q, t)$, scattered by a particle suspension and detected in a far-field position are the measurable effect of the diffusive motion of the particles. The time correlations of these fluctuations are studied in terms of the normalized field autocorrelation function $g(\tau)$, and its expression for a polydisperse cluster system adopts the form [15]

$$
g(\tau)=\frac{\left\langle E(q, 0) E^{*}(q, \tau)\right\rangle}{\langle I(q)\rangle}=\frac{1}{\langle I(q)\rangle} \sum_{n} b_{n}(q) \exp \left(-\Gamma_{n} \tau\right),
$$

where $b_{n}(q)$ contains the relative frequency and the structure of an $n$-particle cluster and $\Gamma_{n}$ is its associated decay rate in which rotational and translational diffusions are included,

$$
\Gamma_{n}=D_{n} q^{2}+6 D_{n}^{r o t},
$$

where $D_{n}$ and $D_{n}^{\text {rot }}$ are the translational and rotational diffusion coefficients of an $n$-particle cluster, assuming that $D_{n}$ and $D_{n}^{r o t}$ are uncoupled. Taking into account the typical values for $q$ and the average cluster size, through its hydrodynamic radius, we can assume that rotational and translational 
diffusions happen in two separated time scales and

$$
D_{n} q^{2} \gg D_{n}^{r o t}
$$

can be considered as a plausible assumption [13].

In order to obtain the average value for the translational diffusion coefficient $\bar{D}(q)$ over the system at any time, the experimental autocorrelation function is treated as an expansion in powers of $\tau$. This treatment is known as cumulant method [16]. The expression for its logarithm results,

$$
\ln \left[g_{\exp }(\tau)\right]=-\kappa_{1} \tau+\frac{1}{2} \kappa_{2} \tau^{2}+\ldots,
$$

where $\kappa_{i}$ is the $i$ th-order cumulant. Relating (5) with (8) and assuming (7), we find that $\kappa_{1}$ represents the average decay rate, $\kappa_{2}$ contains the standard deviation, while $\bar{D}(q)$ is given by

$$
\bar{D}(q)=\frac{\sum_{n} b_{n}(q) D_{n}}{\sum_{n} b_{n}(q)}=\frac{\kappa_{1}}{q^{2}},
$$

which is the basis for the access, through a DLS experiment, to the aggregation kinetics of a particle suspension.

\section{Aggregation kinetics and Smoluchowski's equation}

A time evolution model for the cluster size distribution arising in an aggregating system may be obtained by solving Smoluchowski's rate equation $[17,18]$

$$
\frac{d N_{n}}{d t}=\frac{1}{2} \sum_{i+j=n} k_{i j} N_{i} N_{j}-N_{n} \sum_{i=1}^{\infty} k_{i n} N_{i},
$$

where $N_{n}(t)$ can be used as a frequency distribution in order to obtain the mean value of any function of $n . k_{i j}$ is a set of constants called kernel, hypothetically infinite, in which the whole kinetic information is contained. The aggregation kernel, $k_{i j}$, quantifies the average rate (for all orientational and structural configurations) at which two $i$ - and $j$-particle clusters react and form an $(i+j)$-particle cluster. The above equation, in which fragmentation does not occur, is applied to diluted systems where only binary reactions are contemplated.

A general scheme to classify the aggregation kernels attending their homogeneity was proposed by van Dongen and Ernst [19]. This classification can be expressed as follows:

$$
\begin{gathered}
k_{(a i)(a j)} \sim c^{\lambda} k_{i j},(\lambda \leq 2) \\
k_{i \ll j} \sim i^{\mu} j^{\lambda-\mu},(\lambda-\mu \leq 1),
\end{gathered}
$$

where $c>1$ is a constant, the homogeneity parameter, $\lambda$, correlates the aggregation rates involving two different reactions between similar sized clusters, while $\mu$ controls the rates for the reactions involving different sized clusters. Restrictions over $\lambda$ and $\mu$ come from the nonphysical possibility for which cluster reactivity cannot rise faster than its mass. Restricting ourselves to nongelling kernels $(\lambda \leq 1)$, the scaling theory [20] gives us the asymptotic behavior in time for the number-average cluster size, $\bar{n}_{n}(t)$, as a function of $\lambda$,

$$
\bar{n}_{n}(t)=\frac{\sum_{j} j N_{j}}{\sum_{j} N_{j}} \sim t^{1 /(1-\lambda)} .
$$

On the other hand, the $n$ dependence for the $n$-particle cluster translational diffusion coefficient, $D_{n}$, was estimated assuming the aggregates to be fractal objects [19],

$$
\frac{D_{n}}{D_{0}}=(n)^{-1 / d_{f}}
$$

where $D_{0}$ is the diffusion coefficient of the free individual particles. Considering (12) and (13), the following asymptotic behavior for $\bar{D}(t ; q)$ can be predicted:

$$
\bar{D}(t ; q) \sim t^{-\alpha},
$$

where $\alpha=1 /(1-\lambda) d_{f}$. This behavior is the key to associate a type of kernel to the results obtained for experimental aggregation kinetics.

In order to describe DLCA regime, mentioned in the Introduction, the Brownian kernel is commonly accepted $[17,18]$

$$
k_{i j}^{\text {Brown }}=\frac{1}{4} k_{11}^{\text {Brown }}\left(i^{1 / d_{f}}+j^{1 / d_{f}}\right)\left(i^{-1 / d_{h}}+j^{-1 / d_{h}}\right),
$$

where $k_{11}^{\text {Brown }}$ is the dimer aggregation rate, $d_{f}$ is the previously defined cluster geometric fractal dimension, and $d_{h}$ is the cluster hydrodynamic fractal dimension. In this work, we assume for liposomes $d_{h}=d_{f}$ as other authors do for rigid particles $[21,22]$. In any case, the plausibility for this assumption will be argued later.

A common kind of kernel for describing slow-type regime evolution usually considers the Brownian kernel as a limit, introducing the function $P_{i j}$ (depending on a sticking probability, $\left.p_{11}\right)$ in order to impose a rate of effective contacts [23],

$$
k_{i j}=k_{i j}^{\text {Brown }} P_{i j},\left[P_{i j}=p_{11}(i j)^{\beta}\right] \text {, }
$$

where the probability for an effective monomer-monomer contact, $p_{11}$, has been separated from the total effective combinations of contacts between two $i$ and $j$ clusters, through the $\beta$ parameter, where $\lambda=2 \beta$. $\beta$ lies in general between the limiting values 0 and 0.5 , but an expected value comes from the hypothesis that only particles at the surface of the cluster contribute in the collision [12]. For this multiple contact (MC) diffusionlike case, $\beta$ becomes:

$$
\beta=3\left(d_{f}-d_{f D}\right) / d_{f} d_{f D} .
$$

Here $d_{f}$ is the fractal dimension for the slow aggregates while $d_{f D}$ is the diffusive fractal dimension. As expected, this nondiffusive kernel reduces to the Brownian case when $d_{f}=d_{f D}$. 


\section{MATERIALS AND METHODS}

PS from bovine spinal cord was obtained from Lipid Products (Nutfield, UK). Phospholipid at the proportions indicated below were dissolved in a mixture $(2: 1$, volume ratio) of chloroform and methanol in a round-bottom flask and dried in a rotary evaporator under reduced pressure at $40{ }^{\circ} \mathrm{C}$ to form a thin film on the flask. The film was hydrated with deionized water (MilliQ, Millipore, USA) to give a lipid concentration of $30 \mathrm{mM}$. Multilamellar liposomes (MLV) were formed by constant vortexing for 4 min on a vortex mixer and sonication in a Transonic Digitals bath sonifier (Elma, Germany) for 10 min. MLV were downsized in an Extruder device (Lipex Biomembranes, Canada) through polycarbonate membrane filters of variable pore size under nitrogen pressures of up to $55 \times 10^{5} \mathrm{~N} \mathrm{~m}^{-2}$ [24]. Liposomes were extruded in three steps: first, three consecutive extrusions through a $0.8 \mu \mathrm{m}$ pore diameter filter and three other consecutive extrusions through two stacked $0.4 \mu \mathrm{m}$ membranes. The resulting lipid suspension was then extruded fifteen consecutive times through two stacked $0.2 \mu \mathrm{m}$ filters. After preparation, a nitrogen stream was passed to displace the air, and finally, liposomes were stored at $4-7{ }^{\circ} \mathrm{C}$ in a refrigerator in quiescent conditions until their use.

\section{A. Characterization of the liposomes}

To characterize a liposome suspension, two different requirements are necessary. The first involves their characterization in size and shape while the second concerns the determination of the initial liposome concentration, which is a troublesome problem when vesicles are used as colloidal particles.

In order to determine liposome size, several DLS experiments were performed for different diluted liposome volume fractions and angles, verifying a constant value (in time and experiment) for the mean particle diffusion coefficient, $\bar{D}_{\text {exp }}(t ; q)$, obtained using the cumulant analysis given by (8). The experimental field autocorrelation function results from the scattered intensity autocorrelation function, $g_{I}(\tau)$, through the Siegert relation, $\gamma<1$ being a constant,

$$
g_{I}(\tau)=1+\gamma^{2}\left[g_{\text {field }}(\tau)\right]^{2}
$$

An approximated value for the mean liposome external radius comes from the hydrodynamic radius $R_{h}$ associated to $\bar{D}(t ; q)$ via the Stokes-Einstein relation. Combining these previous DLS experiments with a SLS experiment, an alternative size determination can be done by fitting the form factor, $P(q)$, realizing $\left\langle I_{0}(q)\right\rangle \propto P(q)$. Theoretically, a hollow sphere is usually accepted as a simple geometrical model to describe the form of a liposome. The form factor for a hollow sphere in the context of the Rayleigh-Gans-Debye theory is given by the expression

$$
P(q)=\left[3 \frac{\sin (q R)-q R \cos (q R)-\sin (t q R)+t q R \cos (q R)}{\left(1-t^{3}\right)(q R)^{3}}\right]^{2},
$$

where $R$ is the inner radius of the sphere and $R(t-1)$ the thickness of the shell, a value which when applied to unila-

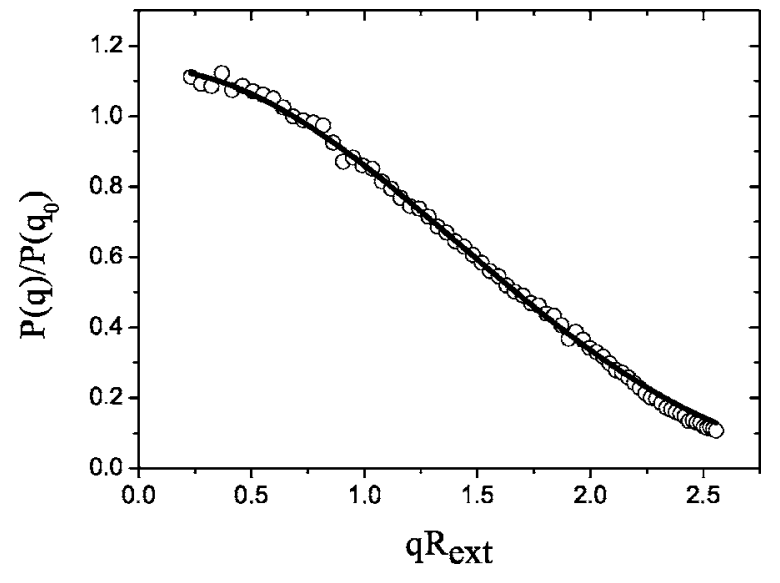

FIG. 1. Normalized form factor $P(q) / P\left(q_{0}\right)$. Open circles stand for experimental values, according to $\left\langle I_{0}(q)\right\rangle \propto P(q)$, whereas solid line denotes the best trimodal fit given by Eq. (20).

mellar liposomes is close to $4.5 \mathrm{~nm}$ [25]. Since a direct estimation for the size distribution in a polydisperse suspension is an ill-conditioned problem, a probability distribution has to be assumed. In order to consider the intrinsic size polydispersity, Schulz's distribution is commonly used as a semiempirical model, where thickness remains constant. This continuous distribution is defined by two parameters, the mean inner diameter, $\bar{\sigma}$, and the relative standard deviation $s$ $[26,27]$. In this work, however, the polydispersity description has been done by using an alternative discrete trimodal distribution, characterized by three modal values and their weights, and related with a Schulz distribution asking for equal values for the five first moments for both distributions (the sixth condition is the normalization condition for the weights). The best estimation comes from the optimum values for $\bar{\sigma}$ and $s$ that minimize mean quadratic error between the linear combination given by the trimodal distribution

$$
P_{t r i}(q)=\sum_{i=1}^{3} a_{i} P_{i}(q)
$$

and the experimental data, where $P_{i}(q)$ is the form factor for a vesicle with modal inner radius $R_{i}$ according with (19), is weighted by $a_{i}$. Figure 1 shows the best fitting for the form factor (normalized by the value corresponding to $30^{\circ}$ ) of the PS vesicles used within this study and its optimum trimodal distribution; the best agreement was reached for $\bar{\sigma}=2 R$ $=184 \mathrm{~nm}$ and $20 \%$ polydispersity. The previous DLS experiments noted above, averaging six individual measures, presented a similar value, $\bar{\sigma}=2 R=179 \mathrm{~nm}$, for the same suspension.

In order to verify the initial liposome concentration, we have followed the procedure by Haro-Perez et al. [28]. If we consider a homogeneous distribution of vesicles in space, we can define a typical distance, $L$, between a pair of neighbour particles associated to a volume fraction $\phi v$,

$$
L=\left[\frac{\frac{4}{3} \pi R_{\mathrm{ext}}^{3}}{\phi v}\right]^{1 / 3},
$$

where $R_{\text {ext }}$ is the mean external radius of the vesicles. 
On the other hand, if the whole PS mass is forming vesicles, the mass fraction, $x$, can be related with $\phi v$ through

$$
\phi v=\frac{x}{\frac{4 \pi\left(R_{\mathrm{ext}}^{3}-R^{3}\right)}{3} \rho^{\prime}} \frac{4 \pi R_{\mathrm{ext}}^{3}}{3},
$$

where $\rho^{\prime}=1.015$ is the relative density PS/water [29].

Although the structure factor contains the whole information of the interparticle distance distribution through its relationship with the pair correlation function, it is useful to give an interpretation in terms of Bragg's law for the interference peak in a nonaggregating and structured dispersion in order to check the expected value for the volume fraction from a known mass fraction. We have obtained the experimental structure factor corresponding to a mass fraction $x=0.0031$, where three individual measures were done at each angle. Bragg's law gives an experimental approach to the most frequent distance, $L$, for a pair of vesicles in the suspension related with a difference in phase, $\Delta \varphi$, for the field intensity scattered by them,

$$
\Delta \varphi=q_{m} L=2 \pi .
$$

The maximum value for $S(q)$ in the previous structure accounts for $q_{m}=0.0120 \mathrm{~nm}^{-1}$, corresponding to $\phi v$ $=0.0219$ using (21) and (23). By means of (22) we obtained $\phi v=0.0227$, where only a relative deviation of $3.5 \%$ comes out.

\section{B. Experiment}

The light-scattering experiments presented in this paper were performed using a slightly modified Malvern 4700 System (UK) working with a $632.8 \mathrm{~nm}$ wavelength $\mathrm{He}-\mathrm{Ne}$ laser. Aggregation was monitored simultaneously by SLS and DLS. For SLS experiments, the photomultiplier arm was previously located at the reference position in order to set the zero angle. After that, the mean scattered intensity was obtained for different angles in the range $10^{\circ}-100^{\circ}$. For DLS experiments, the scattered intensity autocorrelation functions were determined at different times and a fixed angle of $90^{\circ}$ during aggregation and registered by the same computer which controls the instrument. Data analysis was performed using our own computer software.

Aggregation was induced by adding electrolyte to the initially stable aqueous suspensions of liposomes. The water used for sample preparation was purified by inverse osmosis using Millipore equipment. The different electrolyte-particle mixtures were prepared by mixing equal amounts of buffered electrolyte solution and particle suspension through a Y-shaped mixing device in a cylindrical quartz glass cuvette. The electrolyte concentration was varied from $1 \mathrm{mM}$ to $7 \mathrm{mM}$ of $\mathrm{CaCl}_{2}$.

The cluster fractal dimension was obtained by measuring the time-averaged scattered light intensity, $\langle I(q)\rangle$, as a function of the scattering vector. The inequality present in Eq. (3) limits the range for data interpolation, so the correct choice for this range deserves explanatory comments. Such expression is strictly accurate in $1 / R_{\text {aggr }} \ll q \ll 1 / R_{\text {ext }}$ (where $R_{\text {aggr }}$
TABLE I. Experimental kinetic exponents, fractal dimensions, and van Dongen-Ernst homogeneity parameter.

\begin{tabular}{lccc}
\hline \hline$\left[\mathrm{Ca}^{2+}\right](\mathrm{mM})$ & $\alpha$ & $d_{f}$ & $\lambda$ \\
\hline 2.5 & $0.85 \pm 0.03$ & $1.91 \pm 0.07$ & $0.38 \pm 0.04$ \\
3.5 & $0.76 \pm 0.02$ & $1.84 \pm 0.07$ & $0.28 \pm 0.04$ \\
5 & $0.54 \pm 0.02$ & $1.75 \pm 0.06$ & $-0.06 \pm 0.07$ \\
7 & $0.52 \pm 0.02$ & $1.75 \pm 0.05$ & $-0.10 \pm 0.08$ \\
\hline \hline
\end{tabular}

is the radius of the aggregate). However, in practice, a commonly accepted criterion to choose the highest $q$ value is $q_{\max } \leq 1 / R_{\text {ext }}$, whereas the election for the lowest $q$ considers $q_{\min } \geq 1 / R_{\text {aggr }}$. This effective range ensures a nonincursion into Porod and Guinier regimes, respectively [30].

For the DLS results obtained in this work, even in the most unfavourable case $(2.5 \mathrm{mM})$, the ratio $\bar{D}_{\text {exp }} / D_{0}$ is lower than 0.2 (when the fractal regime holds); this implies that in a crude calculus, the corresponding $R_{\text {aggr }}$ reaches about $1000 \mathrm{~nm}$ and $q_{\mathrm{min}} \approx 0.001 \mathrm{~nm}^{-1}$, in accord with the mentioned condition. In our experiments, the smallest value for the modulus of the scattering vector has been, in all cases, $q_{\min } \geq 0.002 \mathrm{~nm}^{-1}$, at which reliability is guaranteed, so a decade in $q$ is covered. A right election for the interpolation interval discards possible misinterpretations between fractal dimensions close to 2 (e.g., see Table I, where $d_{f}=1.91$ at $2.5 \mathrm{mM}$ ) and the exponent at the Guinier regime, which typically operates at $1 / R_{\text {aggr }} \gg q$.

$\langle I(q)\rangle$ measurements were performed for different electrolyte initial concentrations, stopping the aggregation in an advanced stadium by diluting the original mixture under a nonaggregating electrolyte concentration, where a previous DLS experiment was done in order to probe the stabilization in time for the mean diffusion coefficient value. Special attention has to be paid in order to determine the time required by an aggregating system to evolve to a stable state in which its self-similar growth becomes patent, reaching a constant value for the fractal dimension. The necessity to stop the aggregation is obviously imposed by the fact that a complete $I(q)$ scan takes additional time. In consequence, a rigorous measurement protocol has to be chosen. To determine the time at which the SLS scan starts, we made previous experiments, for each sample, stopping the aggregation at different times in order to find out the sufficient time for which a permanent value for the fractal dimensions were verified. With the exception of the $2.5 \mathrm{mM}$ sample, $1 \mathrm{~h}$ resulted in enough in all cases for the initial monomeric concentrations. The results presented within this work were obtained for this self-similar aggregation stadium, according with the commented experimental protocol, averaging $10 I(q)$ scans for each sample.

It is essential to confirm, when we deal with deformable and potentially fusionable particles, the permanence of the individuality, as well as the form, of the initial monomers during the aggregation process. The well-correlated results obtained for the fractal dimension via SLS constitute themselves an indirect confirmation. We have to take into account that according to Eq. (4), the detected signal for the aggre- 

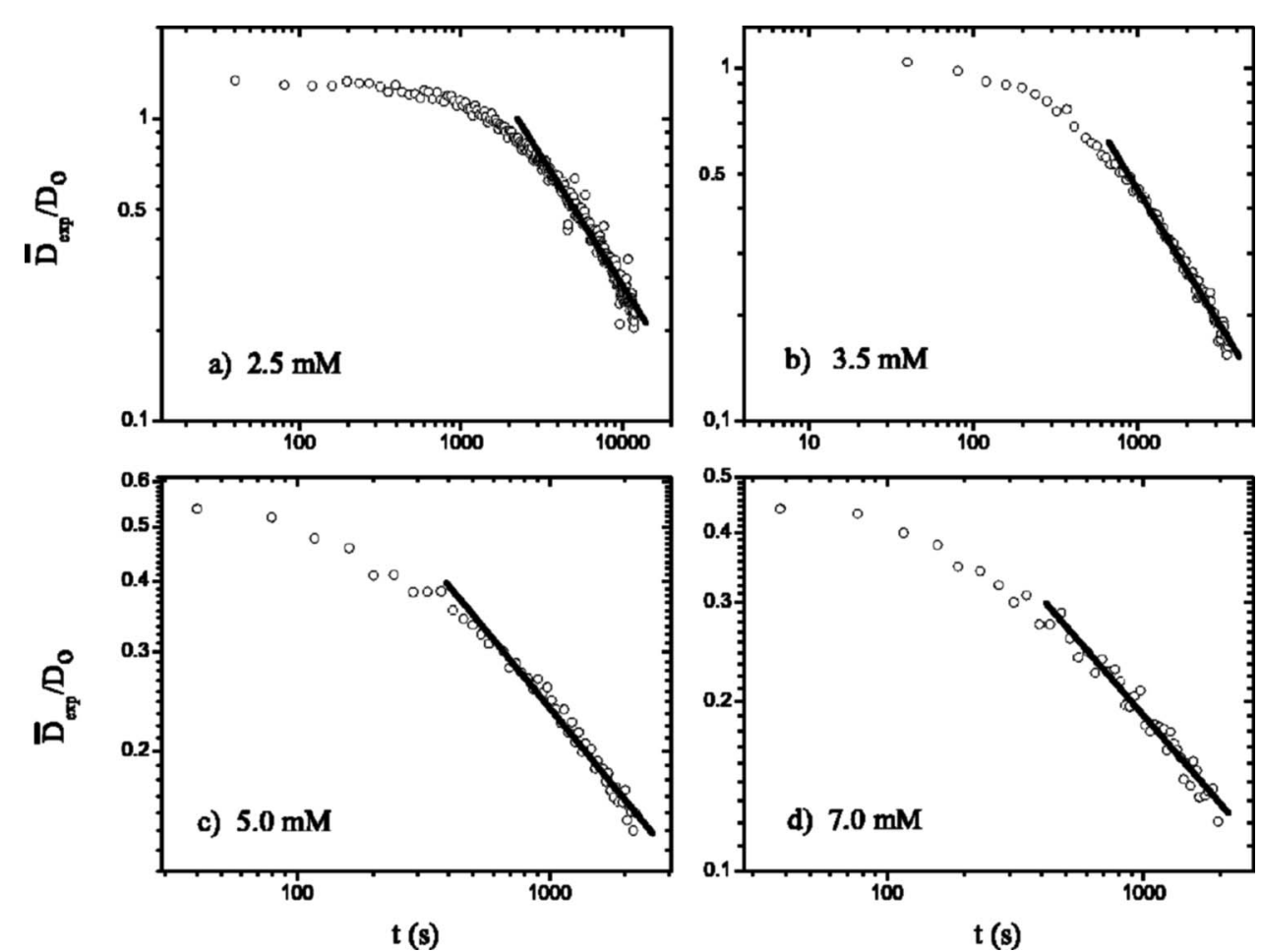

FIG. 2. Normalized experimental mean diffusion coefficient $\bar{D}_{\text {exp }} / D_{0}$ as a function of time for (a) $2.5 \mathrm{mM}$, (b) $3.5 \mathrm{mM}$, (c) $5 \mathrm{mM}$, and (d) $7 \mathrm{mM}$. Open circles stand for experimental values, whereas solid lines denote the asymptotic fit according to (14). gating system is normalized by the detected signal for the diluted system, in which only a monomer information is contained. The resulting signal will have the structural cluster information, with the expected potential behavior, only if the initial monomers are still present in the clusters. In order to have additional evidence, parallel fluorescence experiments, based in the so-called ANTS/DPX assay [31], were performed. No significant reduction in the fluorescent intensity due to the quenching of ANTS by DPX was registered for the electrolyte and particle concentrations used within this work.

\section{RESULTS AND DISCUSSION}

\section{A. Liposome aggregation: Kinetic exponents}

In a preliminary set of experiments, we found that aggregation took place for $\left[\mathrm{CaCl}_{2}\right] \geq 2.5 \mathrm{mM}$. For lower values, neither the diffusion coefficient nor the form factor underwent significant changes. At any rate, similar aggregation salt concentrations were reported by other authors [4]. Apart from that, we have determined the optimal liposome volume fractions to carry out aggregation experiments above this salt concentration, $0.15 \%$ for 2.5 and $3.5 \mathrm{mM}$, and $0.08 \%$ for 5 and $7 \mathrm{mM}$. The liposome volume fraction is a critical parameter from an experimental point of view. It should be chosen to avoid too fast or slow aggregation kinetics.

Time-resolved DLS was employed for monitoring the experimental average diffusion coefficient of the aggregates, $\bar{D}_{\text {exp }}(t ; q)$. In Fig. 2 , the time evolution of $\bar{D}_{\exp }(t ; q)$ is plotted in a double-logarithmic scale for $\left[\mathrm{CaCl}_{2}\right]=2.5,3.5,5$, and $7 \mathrm{mM}$. The curve decreases as time increases and is characterized by a power law at large times. This suggests that the cluster size distribution may be described by the dynamic scaling approach in this time interval. As can be observed, the data align on a straight line even for quite small clusters. This observed power law, $\bar{D}(t ; q) \sim t^{-\alpha}$, is also obtained in all cases. Accordingly the $\alpha$ parameters were obtained by fitting the experimental data. Table I contains the obtained values. It should be pointed out that the values for $5-7 \mathrm{mM}$ are in good agreement with the theoretical prediction given for the diffusion regime $\left(\alpha=1 / d_{f} \approx 0.57\right.$, since $d_{f}=1.75$ is widely accepted for DLCA) [32,33]. DLCA is considered the result of the absence of a repulsive barrier of potential between particles in which aggregation happens at any contact due to an attractive well between the particles, typically operating at very short distances. Regarding the kinetic exponent, $\alpha$, this parameter decreases with increasing the salt concentration, reaching a stable value at $5 \mathrm{mM}$.

\section{B. Liposome aggregation: Fractal dimension}

Although an implicit single scattering assumption is considered in the determination of fractal dimensions via SLS, it is clear that any monomer inside an aggregate scatters a field proceeding from the incident beam radiation in addition to the fields scattered by the rest of the monomers in the cluster. The relevance of this intracluster multiple scattering yields a coupled problem that still remains open. A complete theoretical approach to this problem would involve the size and geometry of the aggregates, as well as the monomers, the optical properties of the scatterers (through their refractive index), and the wavelength of the incident light [30]. An early theoretical work by Chen et al. [34] showed no influence of multiple scattering on the measurement of fractal dimensions [through the behavior of $S(q)$ ] for the range considered in Eq. (4), even for $d_{f}>2$. This theoretical prediction was experimentally confirmed by Lattuada et al. [35] for 

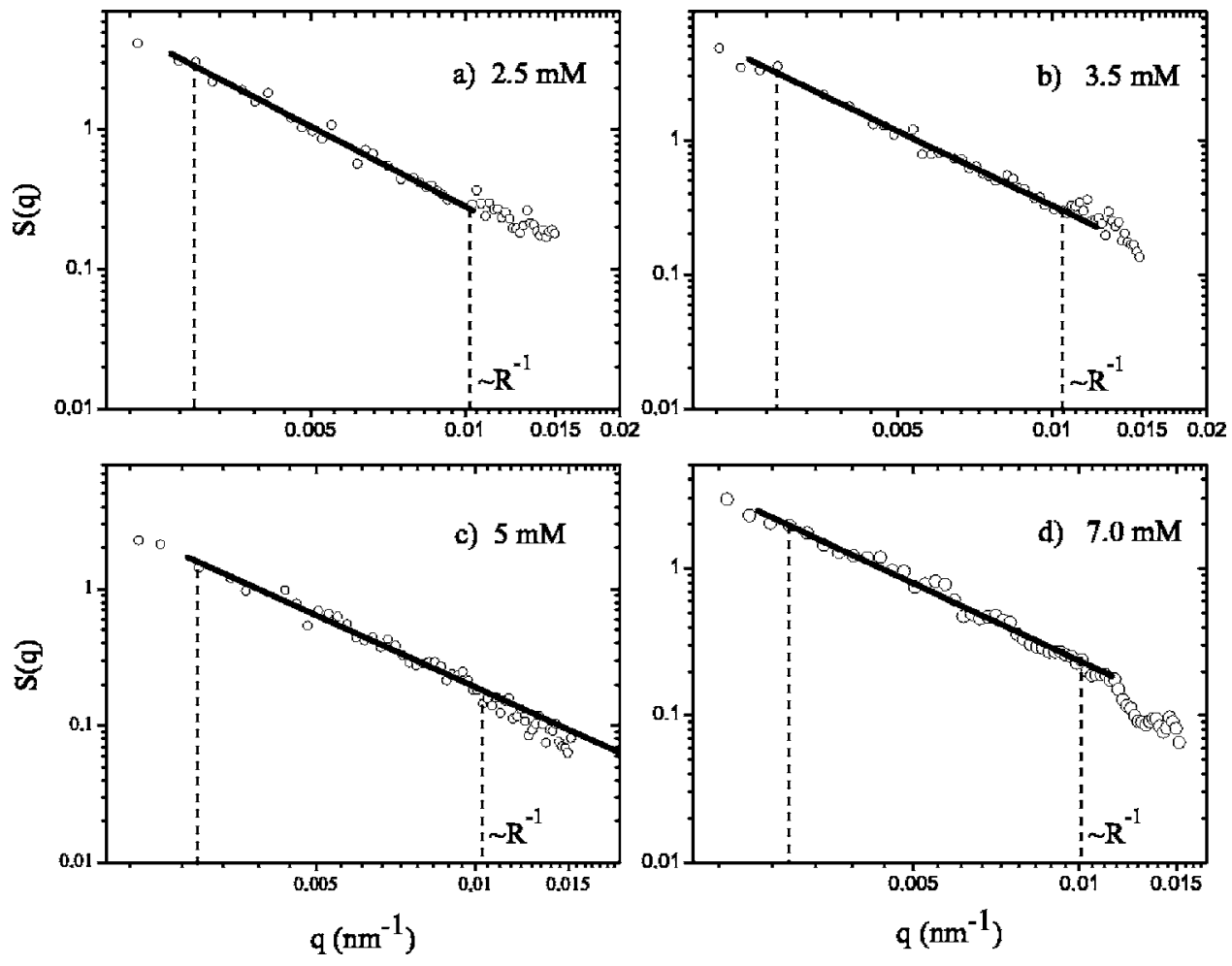

FIG. 3. Structure factor $S(q)$ for (a) $2.5 \mathrm{mM}$, (b) $3.5 \mathrm{mM}$, (c) $5 \mathrm{mM}$, and (d) $7 \mathrm{mM}$. Experimental values (open circles) result from Eq. (4). Solid lines represent the theoretical fit according to the expected behavior given by (3). Vertical dashed lines denote the interpolation $q$ ranges. clusters and monomers comparable in size and refractive index to the particles used within this work $(n=1.36,[36])$. Additionally, the validity of SLS experiments to determine fractal dimensions has been proven for aggregating suspensions of polystyrene latexes, involving large clusters and particles, in which the refractive index of the scatterer particles is considerably greater $(n=1.49)[37,38]$. These experiments confirm the values for the fractal dimension obtained by computer simulations under rapid and slow aggregation regimes. Apart from that, we consider the experimental convergence to a DLCA fractal dimension $\left(d_{f} \approx 1.75\right)$ as an indication of self-consistency for a SLS assay under our suspension conditions, supporting the single scattering assumption.

Having determined the structure factor from lightscattering data [Eq. (4)], $d_{f}$ was obtained by fitting the experimental data to the power law given in Eq. (3). Good agreement was reached in all cases. Figure 3 shows typical plots of the structure factor for $\left[\mathrm{CaCl}_{2}\right]=2.5,3.5,5$, and $7 \mathrm{mM}$. It can be clearly seen that this function presents a functional behavior from which the cluster fractal dimension can be easily obtained. In Table I the fractal dimensions estimated at different electrolyte concentrations are summarized. As can be concluded, the fractal dimension decreases with increasing electrolyte concentration and ranges from 1.91 to 1.75 . It should be noted that the latter fractal dimension is the well-established value for the DLCA regime, so at 5-7 $\mathrm{mM}$ the liposomes have reached such a regime and form ramified open structures. For lower salt concentration, more dense structures will be formed, as the increase in fractal dimension reveals (1.84-1.91). Consequently, SLS experiments provide us a direct access to the aggregate structure and a clear observation of the different aggregation regimes. In a previous work by Lynch et al., a fractal dimen- sion value was reported by fitting the time evolution of turbidity in protein-induced liposome aggregation, although these authors did not analyze the aggregate morphology.

Once the $\alpha$ parameter is known, and using the fractal dimension obtained from independent SLS experiments, the homogeneity $\lambda$ parameter was determined by (14). The results are also summarized in Table I. For the 5 and $7 \mathrm{mM}$, the $\lambda$ value is practically 0 within experimental uncertainty. This is the well-accepted value for DLCA kinetics. The $\lambda$ values between 0.3 and 0.4 point toward a relatively slow aggregation kinetics.

\section{Liposome aggregation: Time evolution of the diffusion coefficient}

The values of fractal dimensions obtained in the preceding section will be used as input in our time evolution model, which involves the resolution of Smoluchowski's equation. As mentioned in the theoretical background, we are assuming $d_{h} \approx d_{f}$. Such assumption is well admitted for hard particles (see, for instance, [21,22]). Although no experimental test has been reported in the same way for liposomes, we have found that the vesicle sizes determined by static light scattering and dynamic light scattering are practically identical, which suggests that the liposome flexibility does not affect the diffusive behavior of the monomers. On the other hand, the fluorescence assays (mentioned above) point to the absence of fusion after aggregation, that is, monomers conserve their individuality inside the aggregates. Additionally, the good correlation for the results of the SLS experiments indicates that the form factor of the monomers appears to be contained in the signal scattered by the aggregating system (as discussed previously). Therefore, in the light of our experiments, liposome aggregates seem to behave as hard ob- 
ject structures (for which this assumption holds).

The experimental values for $\lambda$ and $d_{f}$ obtained above for 5-7 mM suggest a DLCA regime, while a slow regime can be assumed for smaller salt concentration values. At this point we are going to corroborate these features for the whole time evolution with the appropriated kernel model at Smoluchowski's rate equation.

First, we describe the numerical calculation procedure used here. The existence and uniqueness for the solution of Smoluchowski's equation depend on the election for the kernel and the initial condition. For the different proposed kernels, a normalized evolution problem was resolved in terms of the relative concentrations, $X_{n}(T)=N_{n}(T) / N_{1}(0)$, where $T=t\left(N_{1}(0) k_{11} / 2\right), K_{i j}=2 k_{i j} / k_{11}$, and initial monomeric conditions were assumed,

$$
\frac{d X_{n}(T)}{d T}=\frac{1}{2} \sum_{i+j=n} K_{i j} X_{i} X_{j}-X_{n} \sum_{i=1}^{\infty} K_{i n} X_{i},[\vec{X}(0)=(1,0, \ldots)]
$$

In order to obtain the master curve $[\vec{X}(T)]$, which is associated to a $\left\{d_{f}, \lambda\right\}$ input and solution to this dimensionless problem, numerical calculations were carried out by cutting off the number of equations, $N c$, for the previous system, taking under consideration the error related with the noncomputed equations. In order to neglect this cutting-off error, the conservation for the number of monomers was imposed within the whole integration process. This conservation condition can be expressed as

$$
\operatorname{abs}\left(\sum_{i=1}^{N c} i X_{i}(T)-1\right) \leq \varepsilon \approx 0, \forall T,
$$

$\varepsilon$ being the imposed accuracy.

The numerical algorithm was based in a Runge-Kutta fourth-order explicit iterative method.

The estimation for the numerical diffusion coefficient, $\bar{D}_{\text {num }}(t ; q)$, was done according to light-scattering theory in which $\bar{D}(q)$ may be expressed, at any time, as

$$
\bar{D}(q)=\frac{\sum_{n=1}^{N c} b_{n}(q) D_{n}}{\sum_{n=1}^{N c} b_{n}(q)}
$$

where $b_{n}=N_{n} n^{2} S\left(q R_{g}\right)$ and $S\left(q R_{g}\right)$ is the structure factor of an $n$-particle aggregate with radius of gyration $R_{g}$ [15]. In literature, several functional forms for $S\left(q R_{g}\right)$ can be found. The main difficulty lies in obtaining an expression for the structure factor valid for the whole range $q R_{g}>1$. We adopt the expression calculated by Lin et al. [39] directly from computer-generated clusters obtained under diffusion- and reaction-limited conditions.

In order to compare experimental data for the diffusion coefficient $\bar{D}_{\text {exp }}(t ; q)$ with those obtained from the expressions (26) and the solution of (24) for $\bar{D}_{\text {num }}(t ; q)$, a transformation between the normalized time in the numerical model

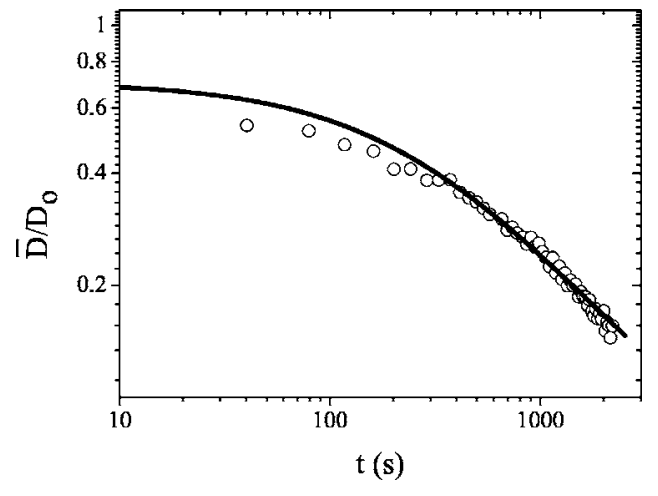

FIG. 4. Normalized mean diffusion coefficient $\bar{D} / D_{0}$ as a function of time $(5 \mathrm{mM})$. Solid line shows the numerical solution for Smoluchowski's equation, assuming the Brownian kernel. Open circles denote the experimental values.

into the real one for the experimental data should be done. The relationship must adopt the form of an afin transformation,

$$
T=m t+n
$$

where $m=N_{1}(0) k_{11} / 2 \equiv t_{\text {agg }}^{-1}, t_{\text {agg }}$ is the so-called aggregation time and $n / m$ gives us the possible delay between the start of the experimental measure and the start of the aggregation process. The values for $m$ and $n$ were determined by imposing the minimum distance between numerical and experimental data through their mean quadratic error over the whole time evolution. This optimization problem takes the form

$$
\min _{m, n} E(m, n)=\min _{m, n} \sum_{i}\left[\bar{D}_{n u m}\left(m t_{i}+n\right)-\bar{D}_{\exp }\left(t_{i}\right)\right]^{2} .
$$

According to the $\lambda$ and $d_{f}$ values obtained, we will analyze the results for $5 \mathrm{mM}$ as a representative case of DLCA regime using the Brownian kernel. Figure 4 shows a good accord between the theoretical curves and the experimental data in the whole time range. This best fitting between numerical and experimental data corresponds to $k_{11}=k_{11}^{\text {Brown }}$ $=(9.3 \pm 1.1) 10^{-20} \mathrm{~m}^{3} \mathrm{~s}^{-1}$. Although this value agrees with those determined by other authors for liposomes [40], it is significantly smaller than the value predicted from Smoluchowski's diffusion model $[17,18]$ and obtained for other systems (latexes). This model assumes that free diffusion works at distances larger or equal than $2 a$ (i.e., geometrical contact). Our $\lambda$ and $d_{f}$ data suggest that this hypothesis is valid at large distances. In our opinion, however, the disagreement mentioned above might be due to the fact that this free diffusion model is not properly working at short distances for liposomes. Although the scope of this paper does not go beyond the geometrical and kinetic properties, this result points to differences in the interaction mechanisms between liposomes and other model systems at short distances.

Now we will investigate a first case of slow aggregation regime $(3.5 \mathrm{mM})$. Figure 5 shows the experimental data and three different theoretical fittings. As expected, the solution obtained using the Brownian kernel is not able to capture the 


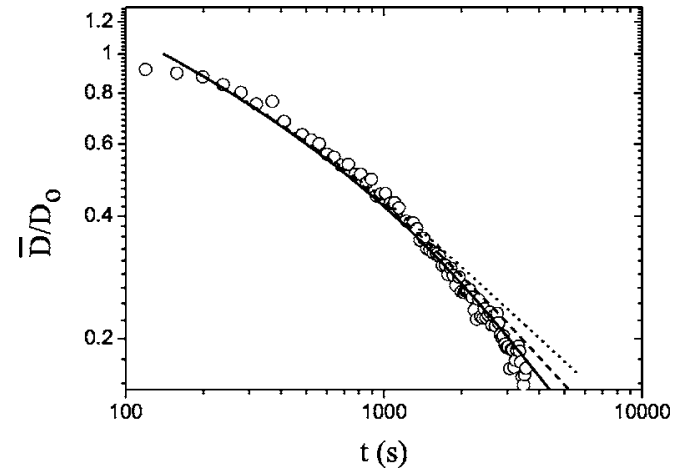

FIG. 5. Normalized mean diffusion coefficient $\bar{D} / D_{0}$ as a function of time $(3.5 \mathrm{mM})$. Three numerical solutions for Smoluchowski's equation are plotted: Dotted line for the Brownian kernel, dashed line for the MC kernel (17), and a solid line for the general slow aggregation kernel (16), using the experimental value obtained for $\beta$. Open circles denote the experimental values.

asymptotic behavior. In the attempt to improve the agreement between experimental data and the theoretical model, the MC kernel given by the expression (17) was applied. Here $d_{f}=1.84$ and $d_{f D}=1.75$. Certain improvement is achieved but at very large times the agreement is still rather poor. Taking into account the general slow aggregation model given by (16) in which $\lambda=2 \beta$ and having experimental access to $\lambda$, we explore if by using this value, a complete description over the whole time range can be done. This is somewhat logical since the experimental $\lambda$ successfully captures the asymptotic behavior. This result (with $\lambda=0.28$ ) is also plotted in Fig. 5. As can be seen, the agreement is considerably improved. In this case, $k_{11}=(3.6 \pm 0.4) 10^{-20} \mathrm{~m}^{3} \mathrm{~s}^{-1}$, which implies $p_{11}=k_{11} / k_{11}^{\text {Brown }}=0.32 \approx 1 / 3$. These quantities are of the order of those reported by other authors practically at the same conditions [41]. $p_{11}$ can also be interpreted in terms of a energy barrier with the help of the Arrhenius equation. In this case, such barrier would be of the order of $(3 / 2) k_{B} T$ (where $k_{B}$ is Boltzmann's constant and $T$ the absolute temperature). In fact, a slow aggregation regime is characterized by a residual repulsive barrier with height compa- rable to the mean kinetic energy of the reacting particles.

Concerning the other slow aggregation $(2.5 \mathrm{mM})$, the same fittings as those mentioned previously were tried. Unfortunately, the accord was not so good. In our opinion, this bad description comes from the possibility of fragmentation, which is not accounted for in Smoluchowski's approach considered here.

\section{CONCLUSIONS}

The aggregation of PS liposome dispersions has been studied in terms of the fractal dimension and the homogeneity parameter of the van Dongen-Ernst. Cluster structure and aggregation kinetics found at $5-7 \mathrm{mM}$ of divalent $\mathrm{Ca}$ indicate that the DLCA regime was reached. For these salt concentrations, the Brownian kernel was able to describe the time evolution of the effective diffusion coefficient. For lower calcium concentrations, the fractal dimension and the homogeneity parameter suggest a transition from DLCA to a slow aggregation regime.

The Brownian kernel does not explain the whole time evolution of the effective diffusion coefficient for the slow kinetics. Although the multiple contact kernel improves the predictions, a slightly modified kernel is required. In this framework, an alternative procedure to determine the dimerization constant has been put forward in both rapid and slow cases. The values here obtained are in good agreement with others reported previously by different methods.

\section{ACKNOWLEDGMENTS}

The authors are grateful to "Ministerio de Educación y Ciencia, Plan Nacional de Investigación, Desarrollo e Innovación Empresa (I+D+i)," Projects MAT2006-12918-C0501, MAT2006-12918-C05-02, and MAT2006-12918-C05-04; the European Regional Development Fund (ERDF); and "Consejería de Innovación, Ciencia y Empresa de la Junta de Andalucía" (Project FQM-392 and incentives for research groups) for financial support. A.M.-M. also thanks the "Programa Ramón y Cajal, 2005, Ministerio de Educación y Ciencia (RYC-2005-000829)."
[1] G. Cevc, Biochim. Biophys. Acta 311, 1031-3 (1990).

[2] Y. Barenholz, Curr. Opin. Colloid Interface Sci. 6, 66 (2001).

[3] S. S. Chrai, R. Murari, and I. Ahmad, Biopharm-the Applied Tech. Biopharm. Development 15, 40 (2002).

[4] S. Nir et al., Prog. Surf. Sci. 3, 1 (1983).

[5] J. Bentz and H. Ellens, Colloids Surf. 30, 65 (1988).

[6] R. Blumenthal et al., Chem. Rev. (Washington, D.C.) 103, 53 (2003).

[7] D. D. Lasic, Handbook of Biological Physics (London, Elsevier, 1995).

[8] J. Wilschut, N. Duzgunes, and D. Papahadjopoulos, Biochemistry 20, 3126 (1981).

[9] F. Bordi et al., Biophys. J. 91, 1513 (2006).

[10] O. Stauch and R. Schubert, Biomacromolecules 3, 565 (2002).
[11] N. J. Lynch, P. K. Kilpatrick, and G. Carbonell, Biotechnol. Bioeng. 50, 151 (1996).

[12] A. Schmitt et al., Phys. Rev. E 62, 8335 (2000).

[13] G. Odriozola et al., J. Colloid Interface Sci. 240, 90 (2001).

[14] T. Vicsek, Fractal Growth Phenomena (World Scientific, Singapore, 1988).

[15] M. Y. Lin et al., J. Phys. Condens. Matter 2, 3093 (1990).

[16] D. E. Koppel, J. Chem. Phys. 57, 4814 (1972).

[17] M. Z. Smoluchowski, Phys. Z. 17, 557 (1916).

[18] M. Z. Smoluchowski, J. Phys. Chem. 92, 129 (1917).

[19] P. G. J. van Dongen and M. H. Ernst, J. Stat. Phys. 50, 295 (1988).

[20] P. G. J. van Dongen and M. H. Ernst, Phys. Rev. A 32, 670 (1985). 
[21] P. Meakin, Z. Y. Chen, and J. M. Deutch, J. Chem. Phys. 82, 3786 (1985).

[22] P. N. Pusey and J. G. Rarity, Mol. Phys. 62, 411 (1987).

[23] M. L. Broide, Ph.D. thesis, Massachusetts Institute of Technology (1988).

[24] M. J. Hope et al., Biochim. Biophys. Acta 812, 55 (1985).

[25] M. T. Roy, M. Gallardo, and J. Estelrich, Bioconjugate Chem. 8, 941 (1997).

[26] B. D'Aguanno and R. Klein, J. Chem. Soc., Faraday Trans. 87, 379 (1991).

[27] B. D'Aguanno and R. Klein, Phys. Rev. A 46, 7652 (1992).

[28] C. Haro-Perez et al., J. Chem. Phys. 119, 628 (2003).

[29] D. D. Lasic, Liposomes: From Physics to Applications (Amsterdam, Elsevier, 1991).

[30] C. M. Sorensen, Aerosol Sci. Technol. 35, 648 (2001).

[31] N. Düzgünes, L. A. Bagatolli, P. Meers, J.-K. Oh, and R. M. Straubinger, Liposomes, A Practical Approach (Oxford Uni- versity Press, 2003).

[32] P. Meakin, Phys. Rev. B 29, 4327 (1984).

[33] M. Kolb, Phys. Rev. Lett. 53, 1653 (1984).

[34] Z. Chen et al., Phys. Rev. B 37, 5232 (1988).

[35] M. Lattuada, H. Wu, and M. Morbidelli, Phys. Rev. E 64, 061404 (2001).

[36] C. Haro-Perez, Ph.D. thesis, Universidad de Granada (2005).

[37] M. Tirado-Miranda, A. Schmitt, J. Callejas-Fernandez, and A. Fernandez-Barbero, Phys. Rev. E 67, 011402 (2003).

[38] M. Tirado-Miranda et al., Colloids Surf., A 162, 67 (2000).

[39] M. Y. Lin et al., Phys. Rev. A 41, 2005 (1990).

[40] S. Nir, J. Wilschut, and J. Bentz, Biochim. Biophys. Acta 668, 275 (1982).

[41] E. P. Day, A. Y. W. Kwok, S. K. Hark, J. T. Ho, W. J. Vail, J. Bentz, and S. Nir, Proc. Natl. Acad. Sci. U.S.A. 77, 4026 (1980). 